\title{
COMPARISON OF REFRACTIVE INDEX OF SHADOW AND DIRECT SUNLIGHT REGION, AND IT'S EFFECT ON LIVING ORGANISM AND PLANTS, THEORETICALLY
}

\author{
Saddam Husain Dhobi, MD Jahangeer Rangrej \\ Department of Physics \\ Tribhuvan University, Kathmandu -44600, 3, Nepal. \\ Innovative Ghar Laboratory \\ Innovative Ghar Nepal, Lalitipur-44700, Nepal
}

\begin{abstract}
This framework developed a mathematical model, to calculate refractive index of shadow region and bright or direct light region, using the parameters such as energy, wavelength, frequency and temperature. After developing the model it help to finding refractive index of the both region, we have compare these refractive index and found that the refractive index of shadow region is greater than that of direct sunlight region. The greater refractive index of shadow region indicate that the energy of the entering photon in shadow region has less energy or energy goes to decrease then before entering into medium or direct sunlight region. Due to this reason the agriculture crops grow in shadow region doesn't get sufficient energy for the growth, but agriculture crops grow in direct sunlight region get sufficient amount of energy to growth. Moreover, such type of problem are face by other living organism and plant in shadow region than that of direct sunlight region, which need more energy.
\end{abstract}

Keywords-Direct Sunlight Region, Energy, Frequency, Refractive Index, Organism and Plants Shadow Region, Wavelength etc.

\section{INTRODUCTION}

Depending on the type of radiation, it is known that the 324 $\mathrm{Wm}^{-2}$ reaching the Earth in the upper atmosphere, $236 \mathrm{Wm}^{-2}$ are reissued into space infrared radiation, $86 \mathrm{Wm}^{-2}$ are reflected by the clouds and $20 \mathrm{Wm}^{-2}$ are reflected by the ground as short-wave radiation. The radiation band corresponds to the visible area with wavelengths between 360 $\mathrm{nm}$ (violet) and $760 \mathrm{~nm}$ (red), it has a great influence on living beings [1].

\author{
Topendra Dangi, Upendra Patel \\ Department of Physics \\ Tribhuvan University, Kathmandu-44600, 3, Nepal.
}

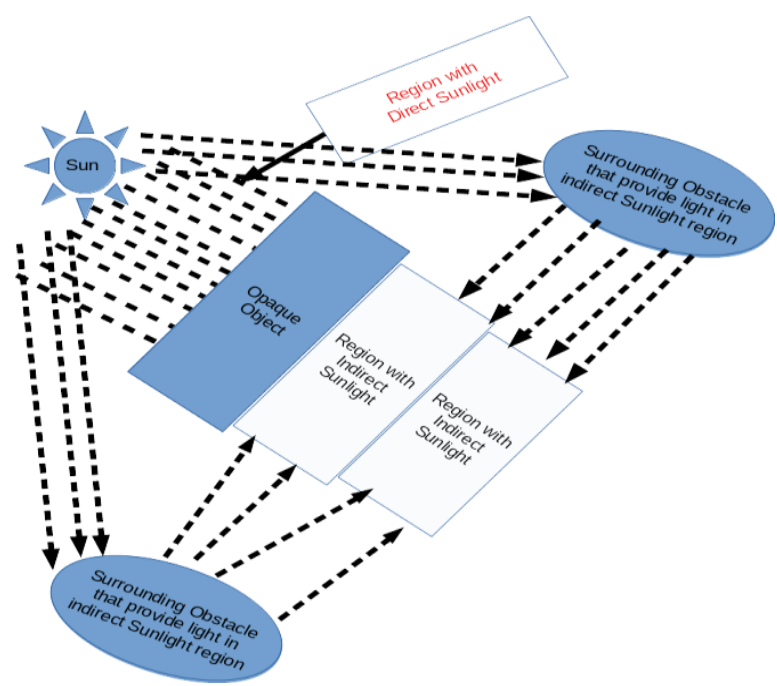

Figure 1: Phenomena of shadow formation and visualization of shadow.

Opaque object and It Effect: Here opaque object is such type of object which have property of blocking the the light which fall on it and formed the shadow behind it. The blocking of light and shadow formation causes the temperature different i.e. the temperature in front of opaque object is greater and causes the density of a air thinner than the side of shadow formed by it. In other word we can also say that the refractive index in front of opaque object, which block the light and formed the shadow, is less than that back or behind the opaque object where shadow is formed. In figure 1 the middle dark green is opaque and behind opaque light white part is shadow formed by opaque object.

Obstacles and It Effect: The obstacle play an important role in the region of shadow formed by opaque object because the energy of sunlight change after strike with it and causes the shadow to visible. When such energy or velocity of light enter in shadow region from bright or direct sunlight region after 


\section{International Journal of Engineering Applied Sciences and Technology, 2019 \\ Vol. 4, Issue 7, ISSN No. 2455-2143, Pages 371-375 \\ Published Online November 2019 in IJEAST (http://www.ijeast.com)}

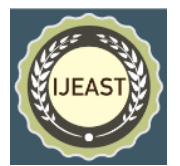

sticking with obstacles the velocity and energy of the sunlight goes to be change due to the deference in the refractive index of the two region i.e. shadow or indirect sunlight region and direct sunlight region. The phenomena of shadow formation and visualization of shadow are shown in figure 1 .

The energy of the particle-wave duality are summarized by the equation $\mathrm{E}=\mathrm{hf}$ where $\mathrm{f}$ is frequency or wavelength $\lambda$, comes in 'packets' or photons, of energy $\mathrm{E}, \mathrm{h}$ is Planck's constant $\left(6.626 \times 10^{-34} \mathrm{Js}\right)$ and $\mathrm{c}$ is the velocity of light $\left(3.00 \times 10^{8} \mathrm{~m} / \mathrm{s}\right)$. Sunlight passing through the earth's atmosphere is attenuated, or reduced, by about $30 \%$ due to Rayleigh scattering, Scattering and Absorption [2], Most energy from sun comes from visible part $\left(\lambda_{\max }=0.5 \mu \mathrm{m}\right)$ most of this energy also reaches the earth surface [3].

\section{REVIEW}

Approximately $99 \%$ of the total solar irradiance $\mathrm{E}_{\mathrm{TSI}}$ is found between $200 \mathrm{~nm}$ and $4000 \mathrm{~nm}$. The range of the photosynthetically available radiation (PAR), i.e. the light that is available to plants for the photosynthesis, is $400 \mathrm{~nm}$ to $700 \mathrm{~nm}$ [4]. The range of visible spectrum: $0.4 \mu \mathrm{m}-0.7 \mu \mathrm{m}$, violet 0.4 $\mu \mathrm{m}-0.446 \mu \mathrm{m}$, blue $0.446 \mu \mathrm{m}-0.5 \mu \mathrm{m}$, green $0.5 \mu \mathrm{m}-0.578$ $\mu \mathrm{m}$, yellow $0.578 \mu \mathrm{m}-0.592 \mu \mathrm{m}$, orange $0.592 \mu \mathrm{m}-0.62 \mu \mathrm{m}$, red $0.62 \mu \mathrm{m}-0.7 \mu \mathrm{m}$, infrared (IR) spectrum: $0.7 \mu \mathrm{m}-100$ $\mu \mathrm{m}$, microwave region: $1 \mathrm{~mm}-1 \mathrm{~m}$ and radio waves: $(>1 \mathrm{~m})$ [5].

The Lorenz-Lorentz equation that the refractive index of a mixture of nonpolar gases is given by the

$\frac{\left(n^{2}-1\right)}{\left(n^{2}+2\right)}=\sum_{i} R_{i} \rho_{i}$

(1)relation

in which $R_{i}$ is the specific refraction and $\rho_{i}$ the partial density of the $i^{\text {th }}$ component of the mixture and $R_{i}=\left[\left(n_{i}^{2}-\right.\right.$ $\left.1) /\left(n_{i}{ }^{2}+2\right)\right]\left(1 / \rho_{i}\right)=\left[4 \pi\left(N_{A} / M_{i}\right) a_{i}\right] / 3$ in which $n_{i}, M_{i}$, and $a_{i}$ are the refractive index at density $\rho_{\mathrm{i}}$, the molecular weight, and the polarizability, respectively, of the $i^{\text {th }}$ component, and $\mathrm{N}_{\mathrm{A}}$ is Avogadro's number. For atmospheric air it is sufficient to write Eq. (1) as the sum of three terms,

$$
\frac{\left(n^{2}-1\right)}{\left(n^{2}+2\right)}=\mathrm{R}_{1} \rho_{1}+\mathrm{R}_{2} \rho_{2}+\mathrm{R}_{3} \rho_{3} .
$$

in which $R_{1}, R_{2}$, and $R_{3}$ are the specific refractions of dry, $\mathrm{CO}_{2}$-free air, of water vapor, and of carbon dioxide, respectively, and $\rho_{1}, \rho_{2}$, and $\rho_{3}$ are the corresponding partial densities [6].

The relation between $\lambda_{\text {vac }}$, the vacuum wavelength, $\lambda_{\text {air }}$, the wavelength in air, and $n$, the refractive index of air, is $\lambda_{\text {vac }}=n$ $\lambda_{\text {air }}[7]$.

The density of moist air, is given by

$$
\mathrm{D}=\frac{\mathrm{PM}_{a}}{\mathrm{RTZ}}\left[1-\left(1-\frac{18.0152}{M_{a}}\right) \frac{\left(\mathrm{Ufe}_{s}\right)}{100 P}\right] .
$$

where $\mathrm{P}$ is the pressure in $\mathrm{P}_{\mathrm{a}}, \mathrm{M}_{\mathrm{a}}$ is the apparent molecular weight of dry air, $\mathrm{R}$ is the universal gas constant, $\mathrm{T}$ is the temperature in kelvins, $\mathrm{Z}$ is the compressibility factor, $\mathrm{U}$ is the relative humidity in percent, and $\mathrm{f}$ is the enhancement factor (a factor which expresses the fact that the effective saturation vapor pressure of water in air is greater than the saturation vapor pressure, $\mathrm{e}_{\mathrm{s}}$, of pure phase over a plane surface of pure ordinary liquid water) [8].

The refractive index or index of refraction (n) is one of the physicochemical properties of substances (optical medium). The refractive index can provide information for us about the behavior of light. Thomas Young was presumably the person who first used, and invented, the name "index of refraction", in 1807. When light passes through the different substances its velocity decreases by increasing of the refractive index of these substances. It can be due to interaction between molecules of components in substrate and effect of these interactions on light. Also in the most substrates, the refractive index decreases by increasing of the temperature. The interaction between molecules decreases as the temperature increases. The refractive index of different substrates measures with refractometers [9].

\section{METHODOLOGY}

Let $n_{\text {direct }}$ be the refractive index of region or medium, where sunlight incidence direct and $\mathrm{n}_{\text {indirect }}$ is the refractive index of a region or medium, where sunlight incidence indirectly (i.e. shadow part) as shown below in figure 2. The region where direct sunlight incidence has more intensity of consider wavelength of light (Visible wavelength of light), while in indirect region the intensity of sunlight is less and is due to secondary light (light after strike with obstacles) as shown in figure 2. The dark blue in center of figure 2 is opaque object and white with slightly blue is shadow part formed by opaque object.

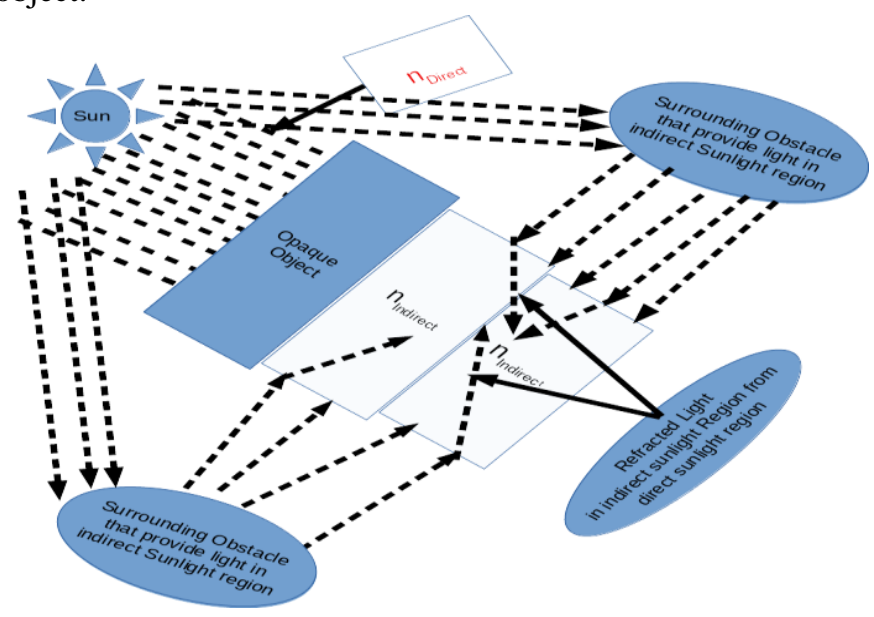

Figure 2: Refraction of light after striking with obstacles and enter from a region having less refractive index to high i.e. direct to indirect sunlight region, and shadow formation phenomena.

Let hf is the energy of light or photon coming from sun in direct region or open space of earth and $\mathrm{hf}_{1}$ is the amount of energy when it collide with obstacles and $\mathrm{hf}_{2}$ is the amount of energy when it enter into the medium where indirect sunlight 


\section{International Journal of Engineering Applied Sciences and Technology, 2019 \\ Vol. 4, Issue 7, ISSN No. 2455-2143, Pages 371-375 \\ Published Online November 2019 in IJEAST (http://www.ijeast.com)}

goes (shadow region) as given figure 3 below. Since we are dealing with shadow (indirect sunlight incidence region) and non-shadow part (direct sunlight incidence region).

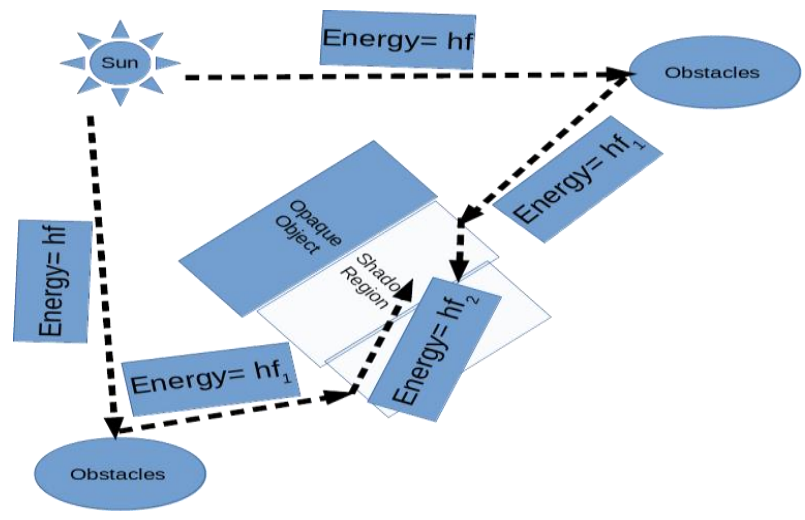

Figure 3: Phenomena of changing energy of a photon or light.

Since refractive index of air is given as

$$
n=\frac{c}{v}
$$

$[10,11]$.

where, $\mathrm{c}=$ velocity of light in vacuum and $\mathrm{v}=$ velocity of light in medium.

In our cases, we are considering the light which enter in shadow region are those light which striking or colliding with surrounding obstacles around shadow region, before entering in shadow region. The energy of light after striking with obstacles is given as

Energy $=h f_{1}$

$$
\text { [from figure 3] }
$$

$$
E_{1}=h f_{1}
$$

where $\mathrm{E}_{1}=$ energy of photon after strike with obstacles, $\mathrm{h}=$ plank constant and $f_{1}=$ frequency of the photon or light after striking the obstacles in a region of temperature $T_{\text {direct }}$.

$$
\begin{array}{r}
E_{1}=\frac{h v_{1}}{\lambda_{1}} \\
{[\text { Since } \mathrm{c}=\lambda \mathrm{f}=>\mathrm{c} / \lambda=\mathrm{f}]}
\end{array}
$$

where $\lambda_{1}=$ wavelength of photon in region after striking whose temperature is $T_{\text {direct }}$ and $c=$ velocity of light in vacuum and $f$ is the frequency of the light in vacuum or same medium.

$$
v_{1}=\frac{E_{1} \lambda_{1}}{h}
$$

For direct sunlight region the refractive index of the medium is $\mathrm{n}_{\text {direct }}$ and given as

$$
n_{\text {direct }}=\frac{c}{v_{1}}
$$

where $\mathrm{v}_{1}=$ velocity of light in the region where sunlight incidence directly.

Putting the value of $\mathrm{v}_{1} n_{\text {direct }}=\frac{c}{\frac{E_{1} \lambda_{1}}{h}}$ from equation (6) in equation (7) we get,

$$
n_{\text {direct }}=\frac{h c}{E_{1} \lambda_{1}} .
$$

Similarly, The energy of light after striking with obstacles and enter into the region or medium where the sunlight goes in indirect is given as

Energy $=h f_{2}$

where $f_{2}=$ frequency of the light after striking with obstacles and entering in shadow or indirect sunlight region.

$$
\begin{array}{r}
E_{2}=h f_{2} \ldots \ldots \ldots \ldots \ldots \\
{[\text { from figure } 3} \\
E_{2}=\frac{h v_{2}}{\lambda_{2}}
\end{array}
$$

$$
\text { [Since } \mathrm{c}=\lambda \mathrm{f}=>\mathrm{c} / \lambda=\mathrm{f}]
$$

where $v_{2}$ and $\lambda_{2}$ is the velocity of light and wavelength in shadow or indirect sunlight region respectively.

$$
v_{2}=\frac{E_{2} \lambda_{2}}{h} .
$$

For this region the refractive index of the medium is $\mathrm{n}_{\text {direct }}$ and given as

$$
n_{\text {indirect }}=\frac{c}{v_{2}}
$$

where $\mathrm{v}_{2}=$ velocity of light in the region where sunlight incidence indirectly (Shadow region).

Putting the value of $v_{2}$ from equation (10) in equation (11) we get

$$
\begin{gathered}
n_{\text {indirect }}=\frac{c}{\frac{E_{2} \lambda_{2}}{h}} \\
n_{\text {indirect }}=\frac{h c}{E_{2} \lambda_{2}} \ldots \ldots \ldots \ldots
\end{gathered}
$$

Now dividing (12) by (8)

$$
\frac{n_{\text {indirect }}}{n_{\text {direct }}}=\frac{\frac{h c}{E_{2} \lambda_{2}}}{\frac{h c}{E_{1} \lambda_{1}}}
$$




$$
\begin{gathered}
\frac{n_{\text {indirect }}}{n_{\text {direct }}}=\frac{h c}{E_{2} \lambda_{2}} \times \frac{E_{1} \lambda_{1}}{h c} \\
\frac{n_{\text {indirect }}}{n_{\text {direct }}}=\frac{E_{1} \lambda_{1}}{E_{2} \lambda_{2}} \\
\frac{n_{\text {indirect }}}{n_{\text {direct }}} \geq 1 \\
n_{\text {indirect }}>n_{\text {direct }} \ldots \ldots \ldots \ldots \ldots .
\end{gathered}
$$

Equation (13) show that the refraction index of the shadow region is greater than that of direct sunlight region because the energy of the light or photon in the shadow region is less than that of direct sunlight region after collided with surrounding obstacles.

Minnaert in his classic Light and Color in the Outdoors gives the following formula

$$
\frac{(n-1)}{n_{0}-1}=\frac{T_{0}}{T}
$$

where $\mathrm{n}_{0}=1.00023$ is the index of refraction of air at an ambient temperature of $\mathrm{T}_{0}=273$ degrees Kelvin and at sea level air pressure. Mirages result from heat gradients above warm surfaces. The temperature above a heated surface usually has an exponential drop off characterized by a surface temperature $\mathrm{T}_{\mathrm{s}}$ and a drop off length $\mathrm{d}_{0}$. For example, the heat field above a hot asphalt road has a surface temperature of approximately 305 degrees Kelvin and a dropoff length, $\mathrm{d}_{0}$, of roughly one centimeter [13].

$$
\begin{aligned}
& n(x)-1 \\
& =\frac{T_{0}\left(n_{0}-1\right)}{T_{0}+\left(T_{s}-T_{0}\right) \exp (-(x-o)) \cdot \frac{n}{d_{0}}}
\end{aligned}
$$

where the plane is defined by its origin o and a normal $n$. The heat field causing the deflections in the left figure has a large drop off $\mathrm{d}_{0}$. In this case the rays are approximately parabolic and we expect the method of Berger et al. to yield good results [14].

Since the refractive index of the medium is depend upon temperature as shown in equation (14) and the more specific in (15) then equation (14) can be used in our situation or case i.e. for direct and indirect sunlight region.

Since $\mathrm{n}_{\text {direct }}$ is the refractive index of direct sunlight region and supposed the temperature in this region is $T_{\text {direct }}$. Similarly, $\mathrm{n}_{\text {indirect }}$ is the refractive index of direct sunlight region and supposed the temperature in this region is $\mathrm{T}_{\text {indirect. Then }}$ equation (14) can be written as

$$
\frac{\left(n_{\text {indirect }}-1\right)}{\left(n_{\text {direct }}-1\right)}=\frac{T_{\text {direct }}}{T_{\text {indirect }}} \text {. }
$$

Since we fill and know the temperature of region where direct sunlight is greater than where indirect sunlight regiion i.e. temperature of shadow region is less than the temperature of direct sunlight region $\left(\mathrm{T}_{\text {direct }}>\mathrm{T}_{\text {indirect }}\right)$.

$$
1<\frac{T_{\text {direct }}}{T_{\text {indirect }}}
$$

Since the temperature of direct sunlight region is greater than that of indirect region.

Then from equation (13) and (14) we get,

$$
\begin{gathered}
1<\frac{T_{\text {direct }}}{T_{\text {indirect }}} \ldots \ldots \ldots \ldots \ldots(18) \\
1<\frac{\left(n_{\text {indirect }}-1\right)}{\left(n_{\text {direct }}-1\right)} \\
\left(n_{\text {direct }}-1\right)<\left(n_{\text {indirect }}-1\right) \\
\left(n_{\text {direct }}\right)<\left(n_{\text {indirect }}\right) \ldots \ldots \ldots \ldots \ldots \ldots \ldots
\end{gathered}
$$

Equation (19) tells us the refractive index of the shadow or indirect sunlight region is greater than that of direct sunlight region because the temperature of the shadow region is less and direct sunlight region is greater.

\section{RESULT AND DISCUSSION}

Equation (19), we show that the refractive index of the shadow region is greater than that of direct sunlight region as the temperature of shadow region is less than that of direct sunlight region. Also from equation (13) we can show that the refractive index of shadow region is greater than that of direct sunlight region as the energy of photon or light in shadow region is less than that of the direct sunlight region i.e. after collided with obstacles.

\section{CONCLUSION}

Hence from above express we can say that the refractive index of shadow region is greater than that direct sunlight region using the parameters like energy, wavelength, frequency and temperature of shadow and direct sunlight region. Moreover, we can suggest the people how the organism and plants get less amount of energy in shadow region which effect on the growth of them.

\section{ACKNOWLEDGMENT:}

We would like to thanks all the member of Innovative Ghar Nepal for providing the research space and peaceful environment during our research study.

\section{REFERENCE}

[1] Campillo, C., Fortes, R., and Prieto, M. D. H. (2012) . Chapter: Solar Radiation Effect on Crop Production, (pp. 168). DOI: 10.5772/34796. 


\section{International Journal of Engineering Applied Sciences and Technology, 2019 \\ Vol. 4, Issue 7, ISSN No. 2455-2143, Pages 371-375 \\ Published Online November 2019 in IJEAST (http://www.ijeast.com)}

[2] (2007). Chapter 1: The Characteristics Of Sunlight, (pp. 1-10).

[3] Eriksson, H. (2015). Introduction to Radiation Theory, (pp31). (Presentation).

[4] Wald, L. (2018) . Lecture Notes: Basics In Solar Radiation At Earth Surface," 1th, MINES ParisTech, PSL Research University, France, (pp. 31-32).

[5] Aggarwal, S. Principles of Remote Sensing, Photogrammetry and Remote Sensing Division: Indian Institute of Remote Sensing, Dehra Dun, (pp. 29).

[6] Owens, J. C. (1967). Optical Refractive Index of Air: Dependence on Pressure, Temperature and Composition, Applied Optics, vol. 6, no. 1, (pp. 51-53).

[7] Jones, F. E. (1981). The Refractivity of Air, Journal Of Research of the National Bureau of Standards, vol. 86, no. 1, (pp. 27).

[8] Jones, F. E. (1978) . The air density equation and the transfer of the mass unit, Journal of Research of the Nattional Bureau of Standards: U.S., vol. 83, no. 5, (pp. 419428).

[9] Chandami, A. S., Choudhari, P. P., and Wadekar, M. P. (2016) . Determination of refractive index, density, molar refraction and Polarizability constant of substituted N,N'-bis (salicyliden)-arylmethanediamines in different binary mixture refractometrically, Advances in Applied Science Research, vol. 7, no. 3, (pp. 120).

[10] Pedrotti, L. S. Module 1.3: Basic Geometrical Optics, Fundamentals of Photonics, (pp. 82).

[11] (2005). TIE-29: Refractive Index and Dispersion, (pp. 1).

[12] Minnaert, M. G. J. (1993) . Light and Color in the Outdoors, Springer Verlag, New York.

[13] Berger, M., Trout, T., and Levit, N. (1990) . Ray Tracing Mirages, IEEE Computer Graphics and Applications, vol. 10 , no. 5, (pp. 36-41). 